\title{
Guest Editorial: Selected Papers from the 13th Brazilian Symposium on Games and Digital Entertainment (SBGames 2014)
}

\section{INTRODUCTION}

Since the first version of SBGames, a huge amount of academic publications related to games and digital entertainment became available. Today SBGames represent an important platform for research in the field. It is an important step for the conference to have a special issue at the Journal on Interactive Systems (JIS).

Selected authors of best papers of the SBGames 2014 Computing Track were invited to submit extended versions of their papers for possible publication, after new rounds of reviews and revisions, at the Journal on Interactive Systems (JIS). As a result, this Special Issue of JIS features five best papers, which were revised and expanded from the conference versions, and underwent a second round of reviews by members of the program committee and additional, outside expert reviewers.

\section{THE ARTICLES}

The first paper, "Combining genetic algorithm and swarm intelligence for task allocation in a real time strategy game", by Anderson R. Tavares, Gianlucca Lodron Zuin, Hector Azpurua, Luiz Chaimowicz, proposes a coordination of multiple agents within a game environment, using a task allocation algorithm based on swarm intelligence and adjust its parameters using a genetic algorithm. The auhors implement their solution using a popular game (StarCraft: BroodWar). The second paper, "Real-Time Screen Space Cartoon Water Rendering with the Iterative Separated Bilateral Filter", by Liordino dos S. Rocha Neto, Antonio L. Apolinario Jr, presents techniques for rendering particle-based liquid simulations that runs in real-time and includes an adjustable performance/quality trade-off. The achieved visual art and style is impressive and can give new visual patterns for modern vide-games. The third paper, "Experimental Approach of the Asymptotic Computational Complexity of Shaders for Mobile Devices with OpenGL ES", by Alex S. C. Lima, Edson A. C. Junior, proposes an experimental approximation of the asymptotic computational complexity of miscellaneous vertex and fragment shaders for Android and iOS platforms. The fourth paper, "BioPlan: Classical Planning with Crowd simulation", by Maurício C. Magnaguagno and Felipe Meneguzzi, describes a crowd simulation experiment that uses classical AI planning to enrich the behavior of agents in the scenario. Using this approach, the authors can express not only the target destination of agents, but also (sub)goals and path preferences. Finally, the fifth paper, "Concept, development and evaluation of a mind action game with the electro-encephalograms as an auxiliary input", by Mark Joselli, Fabio Binder, Esteban Clua and Eduardo Soluri, presents a novel architecture and framework that can help the development of games with both BCI (Brain Computer Interface) and traditional interfaces. As a proof of concept, the paper shows the experience in designing and developing a game prototype using the framework and EEG brainwaves as one of the players input.

We hope you enjoy the selection of papers in this special issue and that they encourage you to address computing issues on digital games in your own work.

\section{ACKNOWLEDGMENTS}

The guest editor of this JIS Special Issue would like to thank the reviewers for their invaluable collaboration and informative reviews, as well as the authors, whose contributions were fundamental to the quality of the papers. We would also like to thank Universidade Federal Fluminense (UFF). Finally, we sincerely thank the JIS Editor-in-Chief, Alberto Raposo, for his support during the best papers review process.

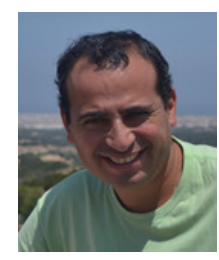

Esteban Walter Gonzales Clua Esteban Walter Gonzales Clua is professor at Universidade Federal Fluminense, coordinator of UFF Medialab and vicedirector of the Computer Science Institute, Young Scientist of the State of Rio in 2009 and 2013. He is graduated in Computer Science by Universidade de São Paulo and has master and doctor degree by PUC-Rio. His main research and development area are Digital Games, Virtual Reality, GPUs and Visualization. He is one of the founders of SBGames (Brazilian Symposium of Games and Digital Entertainment) and was the president of Game Committee of the SBC from 2010 through 2014. Currently, he is the commission member for Brazil at the Technical Committee of Entertainment at the International Federation of Information Processing (IFIP) and honorable member of the board council of ABRAGAMES. In 2015 he was nominated as NVidia Fellow. In 2007 he received the prize from ABRAGAMES as the main contributor from the academia for the development of the game industry at Brazil. $\mathrm{He}$ is the coordinator of the NVIDIA Center of 
Excellence, that is located at the CS Institute of UFF. Esteban belongs to the council of innovation of the Culture Secretary of the State of Rio, is member of the permanent commission of Rio Criativo, Member of the permanent board of innovation and technology at the Legislative board of Rio and member of the Innovation Agency at UFF. 